\title{
Comparison of Different Obesity Indices associated with Type 2 Diabetes Mellitus among Different Sex and Age Groups in China
}

\section{Qiwei Ge}

Nantong University

Min Li

Nantong University

Zhengcheng Xu

Nantong University

Zhigang Qi

Nantong Chongchuan District Center for Disease Control and Prevention

Huiyan Zheng

Nantong Chongchuan District Center for Disease Control and Prevention

\section{Yuxin Cao}

Nantong University

Hao Huang

Nantong University

Xiaoyang Duan

Nantong University

Xun Zhuang ( $\nabla$ ntzhuang@163.com)

Nantong University

\section{Research Article}

Keywords: Obesity, Anthropometric Indices, Type 2 Diabetes Mellitus, Chinese

Posted Date: June 7th, 2021

DOI: https://doi.org/10.21203/rs.3.rs-579184/v1

License: (c) (i) This work is licensed under a Creative Commons Attribution 4.0 International License.

Read Full License 


\section{Abstract}

Background: Obesity is associated with type 2 diabetes mellitus (T2DM), but the obesity index most closely related to type 2 diabetes is still controversial. Therefore, the aim of this study was to compare the associations of five anthropometric indices (body mass index (BMI), body adiposity index (BAI), waist circumference (WC), waist-to-hip ratio (WHR), and waist-to-height ratio (WHtR)) with Type 2 diabetes mellitus (T2DM) among Chinese adults divided into four groups according to sex and age.

Methods: A total of 4,007 adult participants (1,669 males and 2,338 females) were included. Odds ratios (OR) and $95 \%$ confidence intervals were used with binary logistic regression models to estimate the risk of T2DM for each obesity index. Furthermore, we compared the area under the ROC curve (AUC) of each obesity index for the criterion of hypertension under the influence of risk factors.

Results: For the males, WC had the highest value of OR (3.211 and 1.452) and AUC (0.783 and 0.614) in both age groups. However, WHtR $(\mathrm{OR}=2.366, \mathrm{AUC}=0.771)$ and $\mathrm{BMI}(\mathrm{OR}=1.596, \mathrm{AUC}=0.647)$ tended to be the best criteria for T2DM among females in the 18-59 and $\geq 60$ years age groups, respectively.

Conclusions: This study suggests that there is a positive association of obesity-related anthropometric indices with T2DM in different sex and age groups. For males, WC appears to be the best anthropometric index for predicting T2DM. For 18-59 and $\geq 60$ age women, the best obesity indices related with T2DM are WHtR and BMI, respectively.

\section{Background}

Type 2 diabetes mellitus (T2DM), the most common form of diabetes, accounted for more than 96 percent of cases [1]. In China, the prevalence of type 2 diabetes has risen to $11.6 \%$ [2], affecting more than 100 million adults and making it the world's largest number of sufferers [3]. Previous studies have shown that weight gain is an independent risk factor for diabetes, while abdominal obesity is associated with T2DM [4-6]. Anthropometric indices used to define obesity can help in the identification of individuals or groups of people with a variety of specific health thresholds [7]. The commonly used anthropometric indices of obesity are body mass index (BMI), body adiposity index (BAI), waist circumference (WC), waist-to-hip ratio (WHR), and waist-to-height ratio (WHtR) [8].

Some foreign studies have been conducted to assess the relationship between anthropometric obesity indices and T2DM [9-10]; however, the results of these studies are inconsistent. A cross-sectional study in the population of northern Iran showed that while WHR (AUC $=0.7303$ in men, AUC $=0.7529$ in women) had an appropriate discriminatory capability for T2DM, BAI and BMI did not. [11] Another hospital-based cross-sectional study conducted among 363 consecutively selected T2DM showed that WHtR (AUC = $0.85)$ and WC (AUC $=0.79)$ was the best predictor of metabolic syndrome for women and men, respectively [12]. Similar studies have been done in China, but unfortunately the results are not consistent even in the same country. A study of people over the age of 50 in Jinan showed the best indicator of the relationship between obesity and T2DM is WHtR $(O R=2.572)$ for men and BMI $(O R=2.764)$ for women, 
respectively [13]. However, another study in Changchun showed that WHtR (AUC $=0.628$ in men, AUC $=$ 0.676 in women) is the best index to predict T2DM in Chinese adults [14].

The inconsistency of the optimal indicators obtained in the previous studies may be related to ethnic and regional differences [15-18]. In addition, previous studies have stratified studies by age or sex without cross-grouping, and the association between obesity indicators and type 2 diabetes in different sex and age groups remains unclear. Therefore, the aim of this study was to compare the associations of five anthropometric indices (BMI, BAI, WC, WHR, and WHtR) with T2DM among Chinese adults divided into four groups according to sex and age and to supplement similar studies in different regions of China. The thresholds of these anthropometric indices for obesity were also evaluated as a reasonable predictor of T2DM.

\section{Methods}

\section{Study design and participants}

The participants in this study were adult subjects who were enrolled from the baseline survey of the Chronic Disease Study in 2018, which was a cross-sectional study based on urban community-dwelling residents in the Chongchuan district with an estimated 720,000 permanent residents, Nantong City, Jiangsu Province, China.

In the first stage of the enrollment, all 10 blocks of the Chongchuan district were selected and one residential community was randomly selected from each of the blocks. In the second stage, systematic sampling was conducted to select 450 households in each residential community. In the third stage, residents who were older than 18 years and had lived in the district for at least one year were selected from each household. Finally, a total of 12,092 people from 4,500 households were recruited to complete the questionnaires and undergo the relevant physical examinations. 4,035 participants (about 1/3) were randomly selected and fasting blood was drawn to measure plasma blood glucose (FPG). Twenty-eight people were excluded because they provided no information about weight, height, WC, or hip circumference or had a history of type $\otimes$ diabetes, leaving a total of 4,007 participants for the final analyses (Figure.1).

\section{Sample size evaluation}

We calculated the minimum sample size required by using the following formula:

$$
n=z_{1-a / 2}^{2} \times p(1-p) / d^{2} \text { [19]. }
$$




$$
n=400 \times \frac{q}{p}=400 \times \frac{0.9}{0.1}=3600
$$

at $5 \%$ type I error, which is the prevalence of T2DM among Chinese adults, was about 10\% [20]; and d, which is the absolute error or precision, was $10 \%$ in our study. In addition, as the multi-stage random sampling method was used in our study. Therefore, the minimum sample size for our study was 3,600. The final sample size we selected for analysis was large enough compared to the number we calculated.

\section{In this formula, $z_{1-a / 2}^{2}=1.96 \approx 2$}

\section{Data collection}

A trained investigator from the Center for Disease Control and Prevention of the Chongchuan district in Nantong and the School of Public Health in Nantong University interviewed each participant using standardized questionnaires and collected information on demographic data (age, sex, education, etc.), lifestyle behaviors (such as smoking, alcohol consumption, etc.), and family disease history (hypertension, diabetes).

\section{Physical examination}

Anthropometric indices were measured by trained staff who followed standard procedures. Height was measured using a standard stadiometer; each participant underwent the measurement without shoes. Weight was measured to the nearest $0.1 \mathrm{~kg}$ using an electronic scale, with the subjects wearing light indoor clothing. WC was measured to the nearest $0.1 \mathrm{~cm}$ at the midpoint between the lowest rib margin and the level of the anterior superior iliac crest using a flexible anthropometric tape. $\mathrm{HC}$ was measured to the nearest $0.1 \mathrm{~cm}$ at the greatest protrusion of the gluteal muscles. Blood plasma glucose measurements required participants to designate a hospital for measurement on an empty stomach the morning of the test.

This study was in accordance with the World Medical Association Declaration of Helsinki-Ethical Principles for Medical Research Involving Human Subjects and was approved by the Ethics Committee of The Third People's Hospital of Nantong City (No: EK2018009; June 18th, 2018). A written informed consent form was obtained from each participant after they were informed of the objectives and benefits of this study.

\section{Definitions of variables}


Patients who had type 2 diabetes mellitus were defined as those who had been diagnosed in a secondary hospital or who had FPG $\geq 7.0 \mathrm{mmol} / \mathrm{L}$ in the fasting blood glucose testing [21]. BMI was calculated as weight $(\mathrm{kg})$ divided by the square of height $\left(\mathrm{m}^{2}\right)$. According to "the standards of the Chinese guidelines for the prevention and control of overweight and obesity in adults," BMl $\geq 24 \mathrm{~kg} / \mathrm{m}^{2}$ and $\geq 28 \mathrm{~kg} / \mathrm{m}^{2}$ was regarded as being overweight and obese, respectively [22]. Body adiposity index (BAl) was measured using the formula [hip circumference $(\mathrm{cm}) /$ height $^{1.5}(\mathrm{~m})$ ] $-18, \mathrm{BAl} \geq 25.5 \mathrm{~kg} / \mathrm{m}^{2} \& \geq 29.0 \mathrm{~kg} / \mathrm{m}^{2}$ for males, $\geq 30.5 \& \geq 35.0$ for females was regarded as being overweight and obese [23]. WC $\geq 90 \mathrm{~cm}$ for males and $\geq 80 \mathrm{~cm}$ for females were defined central obesity. WHR was calculated as WC $(\mathrm{cm})$ divided by $\mathrm{HC}(\mathrm{cm})$, with values $>0.9$ for males and $>0.8$ for females indicating central obesity. WHtR was calculated as WC $(\mathrm{cm})$ divided by height $(\mathrm{cm})$, and values $\geq 0.5$ indicated obesity [22].

\section{Statistical Analyses}

All quantitative variables were described as mean and standard deviation (SD) for normal distributions, whereas qualitative data were described as percentages. The student $t$ test and chi-square test were used to estimate the difference between quantitative and qualitative data of different groups, respectively. The following weight variables were estimated in this study: BMI, BAl, WC, WHR, and WHtR. Odds ratios (OR) and $95 \%$ confidence intervals $(95 \% \mathrm{Cl})$ were used with binary logistic regression models to estimate the risk of T2DM for each obesity index.

For each index, receiver operating characteristic (ROC) curve analysis was used to identify the value with the Youden index as a predictor of T2DM. $P$ values $<0.05$ were considered significant. The statistical analyses were performed using SPSS 24.0 for Windows.

\section{Results}

The demographic data for the 12,064 eligible participants included in this study are shown in Table 1. The mean age of the participants was $61.78 \pm 14.95$ years, and at the age of 60 , the population was divided into two groups. Naturally, the prevalence of T2DM increased with increasing age. The ratio of male to female participants was 1:0.71, and males had a higher risk of T2DM than females. Regarding education, participants who had primary school or lower education accounted for the highest percentage (44.1 percent). The higher the education level, the lower the risk of T2DM. In addition, smoking status, alcohol drinking status, positive family history of diabetes and hypertension were significantly correlated with T2DM. Finally, there was a statistically significant difference between participants who had T2DM and those who did not with BMI, WC and WHtR. 
Table 1

Sociodemographic and Lifestyle Characteristics distribution among participants

\begin{tabular}{|c|c|c|c|c|c|}
\hline Variable & $\begin{array}{l}\text { Total }(n= \\
\text { 4007) }\end{array}$ & $\begin{array}{l}\text { No diabetes }(n= \\
3376)\end{array}$ & $\begin{array}{l}\text { Diabetes }(n= \\
631)\end{array}$ & $t / \mathrm{OR}(95 \% \mathrm{Cl})$ & $\mathbf{P}$ \\
\hline Age & $\begin{array}{l}61.78 \pm \\
14.95\end{array}$ & $60.36 \pm 15.34$ & $69.4 \pm 9.54$ & & $\begin{array}{l}< \\
0.001\end{array}$ \\
\hline $18-$ & $3376(84.3)$ & $1390(94.0)$ & 1986(78.5) & 1 & \\
\hline $60-$ & 631(15.7) & $88(6.0)$ & $543(21.5)$ & $\begin{array}{l}4.319(3.413- \\
5.465)\end{array}$ & $\begin{array}{l}< \\
0.001\end{array}$ \\
\hline \multicolumn{6}{|l|}{ Sex } \\
\hline Male & 1669(41.7) & 1382(40.9) & $287(45.5)$ & 1 & \\
\hline Female & 2338(58.3) & 1994(59.1) & $344(54.5)$ & $\begin{array}{l}0.831(0.700- \\
0.986)\end{array}$ & 0.034 \\
\hline \multicolumn{6}{|l|}{ Education } \\
\hline $\begin{array}{l}\text { Primary school and } \\
\text { lower }\end{array}$ & 1236(30.8) & $958(28.4)$ & $278(44.1)$ & 1 & \\
\hline Secondary school & $1252(31.2)$ & 1050(31.1) & $202(32.0)$ & $\begin{array}{l}0.663(0.542- \\
0.811)\end{array}$ & $\begin{array}{l}<.001 \\
0.00\end{array}$ \\
\hline $\begin{array}{l}\text { High secondary } \\
\text { school }\end{array}$ & 838(20.9) & $727(21.5)$ & 111(17.6) & $\begin{array}{l}0.526(0.414- \\
0.669)\end{array}$ & $\begin{array}{l}< \\
0.001\end{array}$ \\
\hline $\begin{array}{l}\text { College or } \\
\text { university }\end{array}$ & $386(9.6)$ & $360(10.7)$ & $26(4.1)$ & $\begin{array}{l}0.249(0.164- \\
0.379)\end{array}$ & $\begin{array}{l}< \\
0.001\end{array}$ \\
\hline $\begin{array}{l}\text { Bachelor and } \\
\text { higher }\end{array}$ & $295(7.4)$ & $281(8.3)$ & $14(2.2)$ & $\begin{array}{l}0.172(0.099- \\
0.298)\end{array}$ & <. \\
\hline \multicolumn{6}{|l|}{ Smoking } \\
\hline No & $3479(86.8)$ & 2938(87.0) & $541(85.7)$ & 1 & \\
\hline Yes & $446(11.1)$ & $380(11.3)$ & $66(10.5)$ & $\begin{array}{l}0.943(0.715- \\
1.244)\end{array}$ & 0.679 \\
\hline Quit & $82(2.0)$ & $58(1.7)$ & $24(3.8)$ & $\begin{array}{l}2.247(1.384- \\
3.648)\end{array}$ & 0.001 \\
\hline \multicolumn{6}{|l|}{ Alcohol drinking } \\
\hline No & $3224(80.5)$ & 2723(80.7) & $501(79.4)$ & 1 & \\
\hline Yes & $726(18.1)$ & 612(18.1) & $114(18.1)$ & $\begin{array}{l}1.012(0.811- \\
1.263)\end{array}$ & 0.913 \\
\hline
\end{tabular}

$p<0: 05$, statistically significant. 


\begin{tabular}{|c|c|c|c|c|c|}
\hline Variable & $\begin{array}{l}\text { Total }(n= \\
\text { 4007) }\end{array}$ & $\begin{array}{l}\text { No diabetes }(n= \\
3376)\end{array}$ & $\begin{array}{l}\text { Diabetes }(n= \\
631)\end{array}$ & $t / \mathrm{OR}(95 \% \mathrm{Cl})$ & $\mathbf{P}$ \\
\hline Quit & $57(1.4)$ & $41(1.2)$ & $16(2.5)$ & $\begin{array}{l}2.121(1.181- \\
3.809)\end{array}$ & 0.012 \\
\hline \multicolumn{6}{|l|}{ Sweet tooth } \\
\hline No & $3844(95.9)$ & 3232(95.7) & 612(97.0) & 1 & \\
\hline Yes & $163(4.1)$ & $144(4.3)$ & $19(3.0)$ & $\begin{array}{l}0.697(0.429- \\
1.133)\end{array}$ & 0.145 \\
\hline \multicolumn{6}{|l|}{ Physical activity } \\
\hline No & 1969(49.1) & 1658(49.1) & $311(49.3)$ & 1 & \\
\hline Yes & 2038(50.9) & 1718(50.9) & $320(50.7)$ & $\begin{array}{l}0.993(0.838- \\
1.177)\end{array}$ & 0.935 \\
\hline \multicolumn{6}{|l|}{ Family history } \\
\hline No & $3708(92.5)$ & 3192(94.5) & $516(81.8)$ & 1 & \\
\hline Yes & $299(7.5)$ & $184(5.5)$ & 115(18.2) & $\begin{array}{l}3.866(3.008- \\
4.969)\end{array}$ & $\begin{array}{l}<.001 \\
0.001\end{array}$ \\
\hline \multicolumn{6}{|l|}{ Hypertension } \\
\hline No & 2721(67.9) & $2360(69.9)$ & $361(57.2)$ & 1 & \\
\hline Yes & 1286(32.1) & 1016(30.1) & $270(42.8)$ & $\begin{array}{l}1.737(1.422- \\
2.054)\end{array}$ & $\begin{array}{l}< \\
0.001\end{array}$ \\
\hline \multicolumn{6}{|l|}{$\mathrm{BMI}, \mathrm{kg} / \mathrm{m}^{2}$} \\
\hline Normal & 2064(51.5) & $1801(53.5)$ & $258(41.0)$ & 1 & \\
\hline Overweight/obesity & 1941(48.5) & $1570(46.5)$ & $371(59.0)$ & $\begin{array}{l}1.650(1.388- \\
1.961)\end{array}$ & $\begin{array}{l}<.001 \\
0.001\end{array}$ \\
\hline \multicolumn{6}{|l|}{ WC, $\mathrm{cm}$} \\
\hline Normal & $1829(45.6)$ & $1603(47.5)$ & $226(35.8)$ & 1 & \\
\hline Overweight/obesity & $2178(54.4)$ & $1773(52.5)$ & $405(64.2)$ & $\begin{array}{l}1.620(1.358- \\
1.932)\end{array}$ & $\begin{array}{l}< \\
0.001\end{array}$ \\
\hline \multicolumn{6}{|l|}{ BAl } \\
\hline Normal & 2591(64.7) & 2253(66.7) & $338(53.6)$ & 1 & \\
\hline Overweight/obesity & 1416(35.3) & 1123(33.3) & 293(46.4) & $\begin{array}{l}1.739(1.464- \\
2.066)\end{array}$ & $\dot{0.001}$ \\
\hline
\end{tabular}

$p<0: 05$, statistically significant. 


\begin{tabular}{|c|c|c|c|c|c|}
\hline Variable & $\begin{array}{l}\text { Total }(n= \\
\text { 4007) }\end{array}$ & $\begin{array}{l}\text { No diabetes }(n= \\
3376)\end{array}$ & $\begin{array}{l}\text { Diabetes }(n= \\
631)\end{array}$ & $t /$ OR $(95 \% \mathrm{Cl})$ & $\mathbf{P}$ \\
\hline \multicolumn{6}{|l|}{ WHR } \\
\hline Normal & $972(24.3)$ & $831(24.6)$ & $141(22.3)$ & 1 & \\
\hline Overweight/obesity & $3035(75.7)$ & $2545(75.4)$ & 490(77.7) & $\begin{array}{l}1.135(0.926- \\
1.390)\end{array}$ & 0.222 \\
\hline \multicolumn{6}{|l|}{ WHtR } \\
\hline Normal & $2528(63.1)$ & 2206(65.3) & $322(51.0)$ & 1 & \\
\hline Overweight/obesity & 1479(36.9) & 1170(34.7) & $309(49.0)$ & $\begin{array}{l}1.809(1.524- \\
2.148)\end{array}$ & $<.001$ \\
\hline
\end{tabular}

Considering the confounding effect of age and sex, we divided the participants into four subgroups based both variables (Table 2). In all four subgroups, people who had T2DM had higher WC than the controls $(P<0.05)$ and WHR was not significantly different between the T2DM group and the controls ( $P$ $>0.05$ ). However, for males, BMI was not significantly different between the T2DM group and the controls $(P>0.05)$, whereas in the female groups, BMI was higher in the T2DM group. Moreover, BAI and WHtR was higher in the T2DM group excluding the male $\geq 60$ age group. 
Table 2

Anthropometric measurement characteristics of participants

\begin{tabular}{|c|c|c|c|c|c|c|c|}
\hline Variable & $\begin{array}{l}\text { No } \\
\text { T2DM }\end{array}$ & T2DM & $P$ & Variable & $\begin{array}{l}\text { No } \\
\text { T2DM }\end{array}$ & T2DM & $P$ \\
\hline $\begin{array}{l}\text { Male } \\
\text { (age:18-59) }\end{array}$ & & & & $\begin{array}{l}\text { Female } \\
\text { (age:45-59) }\end{array}$ & & & \\
\hline BMI (kg/m2) & $\begin{array}{l}24.11 \pm \\
2.72\end{array}$ & $\begin{array}{l}25.78 \pm \\
2.92\end{array}$ & 0.053 & BMI (kg/m2) & $\begin{array}{l}23.21 \pm \\
2.90\end{array}$ & $\begin{array}{l}25.06 \pm \\
3.19\end{array}$ & $<.001$ \\
\hline BAl & $\begin{array}{l}25.01 \pm \\
4.50\end{array}$ & $\begin{array}{l}27.26 \pm \\
4.86\end{array}$ & 0.048 & BAI & $\begin{array}{l}26.62 \pm \\
4.27\end{array}$ & $\begin{array}{l}29.28 \pm \\
3.60\end{array}$ & $\begin{array}{l}< \\
0.001\end{array}$ \\
\hline WC (cm) & $\begin{array}{l}85.71 \pm \\
7.90\end{array}$ & $\begin{array}{l}88.74 \pm \\
7.84\end{array}$ & 0.021 & WC (cm) & $\begin{array}{l}80.14 \pm \\
6.63\end{array}$ & $\begin{array}{l}84.53 \pm \\
7.87\end{array}$ & $\begin{array}{l}< \\
0.001\end{array}$ \\
\hline WHR & $\begin{array}{l}0.89 \pm \\
0.06\end{array}$ & $\begin{array}{l}0.88 \pm \\
0.06\end{array}$ & 0.591 & WHR & $\begin{array}{l}0.87 \pm \\
0.06\end{array}$ & $\begin{array}{l}0.87 \pm \\
0.05\end{array}$ & 0.990 \\
\hline WHtR & $\begin{array}{l}0.49 \pm \\
0.05\end{array}$ & $\begin{array}{l}0.52 \pm \\
0.04\end{array}$ & 0.001 & WHtR & $\begin{array}{l}0.49 \pm \\
0.04\end{array}$ & $\begin{array}{l}0.52 \pm \\
0.05\end{array}$ & $<.001$ \\
\hline $\begin{array}{l}\text { Male (age: } \\
\geq 60 \text { ) }\end{array}$ & & & & $\begin{array}{l}\text { Female (age: } \\
\geq 60 \text { ) }\end{array}$ & & & \\
\hline $\mathrm{BMI}(\mathrm{kg} / \mathrm{m} 2)$ & $\begin{array}{l}24.27 \pm \\
3.39\end{array}$ & $\begin{array}{l}24.66 \pm \\
3.32\end{array}$ & 0.112 & $\mathrm{BMI}(\mathrm{kg} / \mathrm{m} 2)$ & $\begin{array}{l}24.12 \pm \\
3.14\end{array}$ & $\begin{array}{l}25.18 \pm \\
4.16\end{array}$ & $\begin{array}{l}< \\
0.001\end{array}$ \\
\hline BAl & $\begin{array}{l}25.45 \pm \\
5.43\end{array}$ & $\begin{array}{l}25.61 \pm \\
4.83\end{array}$ & 0.536 & BAl & $\begin{array}{l}29.08 \pm \\
4.83\end{array}$ & $\begin{array}{l}30.09 \pm \\
4.86\end{array}$ & 0.001 \\
\hline WC (cm) & $\begin{array}{l}86.54 \pm \\
7.83\end{array}$ & $\begin{array}{l}87.84 \pm \\
8.52\end{array}$ & 0.033 & WC (cm) & $\begin{array}{l}83.46 \pm \\
8.08\end{array}$ & $\begin{array}{l}85.48 \pm \\
7.84\end{array}$ & $\begin{array}{l}< \\
0.001\end{array}$ \\
\hline WHR & $\begin{array}{l}0.90 \pm \\
0.05\end{array}$ & $\begin{array}{l}0.91 \pm \\
0.13\end{array}$ & 0.147 & WHR & $\begin{array}{l}0.88 \pm \\
0.06\end{array}$ & $\begin{array}{l}0.89 \pm \\
0.10\end{array}$ & 0.334 \\
\hline WHtR & $\begin{array}{l}0.51 \pm \\
0.05\end{array}$ & $\begin{array}{l}0.51 \pm \\
0.05\end{array}$ & 0.052 & WHtR & $\begin{array}{l}0.52 \pm \\
0.05\end{array}$ & $\begin{array}{l}0.54 \pm \\
0.05\end{array}$ & $<.001$ \\
\hline \multicolumn{8}{|c|}{$\begin{array}{l}\text { BMI, body mass index; BAl, body adiposity index; WC, waist circumference; WHR, waist-to-hip ratio; } \\
\text { WHtR, waist-to-height ratio. }\end{array}$} \\
\hline \multicolumn{8}{|c|}{ Values are presented as mean \pm SD. } \\
\hline $\mathrm{p}<0.05$, stati & Ily sign & & & & & & \\
\hline
\end{tabular}

The associations between the prevalence of T2DM and the obesity indices are shown in Table 3. In both groups of females, the findings indicated positive associations between the five obesity indices but WHR with T2DM even after adjustments for risk-related factors in Model 2. The difference was that WHtR (2.366) had the highest OR value in the 18-59 age group, while BMI (1.596) was the highest in the $\geq 60$ age group after adjusting for risk factors. However, the associations in the male groups compared with the female groups were completely different. In the male aged 18-59 group, BAl, WC and WHtR were positively associated with T2DM, and the highest value of OR (3.211) was WC in Model 2. While in the 
male aged $\geq 60$ group, only WC had positive associations with T2DM and the value of OR was 1.478 (Model 1) and 1.452 (Model 2).

Table 3

Crude and Adjusted ORs for T2DM in Relation to Anthropometric Indices

\begin{tabular}{|c|c|c|c|c|c|}
\hline Variable & $\begin{array}{l}\text { Model } 1 \text { OR } \\
(95 \% \mathrm{Cl})\end{array}$ & $\begin{array}{l}\text { Model } 2 \text { OR } \\
(95 \% \mathrm{Cl})\end{array}$ & Variable & $\begin{array}{l}\text { Model } 1 \text { OR } \\
(95 \% \mathrm{Cl})\end{array}$ & $\begin{array}{l}\text { Model } 2 \text { OR } \\
(95 \% \mathrm{Cl})\end{array}$ \\
\hline $\begin{array}{l}\text { Male } \\
\text { (age:18- } \\
59)\end{array}$ & & & $\begin{array}{l}\text { Female } \\
\text { (age:18-59) }\end{array}$ & & \\
\hline $\begin{array}{l}\mathrm{BMI}(\geq 24 \\
\mathrm{kg} / \mathrm{m} 2)\end{array}$ & $\begin{array}{l}1.492(0.780- \\
2.855)\end{array}$ & $\begin{array}{l}1.540(0.782- \\
3.032)\end{array}$ & $\begin{array}{l}\mathrm{BMI}(\geq 24 \\
\mathrm{kg} / \mathrm{m} 2)\end{array}$ & $\begin{array}{l}3.176(1.733- \\
5.819) \star \star\end{array}$ & $\begin{array}{l}2.183(1.158- \\
4.113) \text { * }\end{array}$ \\
\hline $\begin{array}{l}\text { BAl }(\geq \\
25.5)\end{array}$ & $\begin{array}{l}2.184(1.146- \\
4.161) \star\end{array}$ & $\begin{array}{l}2.178(1.112- \\
4.267) \star\end{array}$ & $\mathrm{BAI}(\geq 30.5)$ & $\begin{array}{l}2.162(1.127- \\
4.148) *\end{array}$ & $\begin{array}{l}1.508(1.059- \\
2.998) *\end{array}$ \\
\hline $\begin{array}{l}\mathrm{WC}(\geq 90 \\
\mathrm{cm})\end{array}$ & $\begin{array}{l}3.699(1.912- \\
7.156) * \star\end{array}$ & $\begin{array}{l}3.211(1.625- \\
6.346) \star \star\end{array}$ & $\begin{array}{l}\text { WC }(\geq 80 \\
\mathrm{cm})\end{array}$ & $\begin{array}{l}2.849(1.360- \\
5.969) *\end{array}$ & $\begin{array}{l}2.287(1.071- \\
4.883) *\end{array}$ \\
\hline $\begin{array}{l}\text { WHR (> } \\
0.9)\end{array}$ & $\begin{array}{l}0.900(0.475- \\
1.704)\end{array}$ & $\begin{array}{l}0.890(0.455- \\
1.740)\end{array}$ & WHR $(>0.8)$ & $\begin{array}{l}1.074(0.415- \\
2.783)\end{array}$ & $\begin{array}{l}0.814(0.306- \\
2.168)\end{array}$ \\
\hline $\begin{array}{l}\text { WHtR }(\geq \\
0.5)\end{array}$ & $\begin{array}{l}3.189(1.676- \\
6.068) * \star\end{array}$ & $\begin{array}{l}2.827(1.446- \\
5.527) \star\end{array}$ & $\mathrm{WHtR}(\geq 0.5)$ & $\begin{array}{l}3.480(1.919- \\
6.312) \star \star\end{array}$ & $\begin{array}{l}2.366(1.265- \\
4.427) *\end{array}$ \\
\hline $\begin{array}{l}\text { Male (age: } \\
\geq 60 \text { ) }\end{array}$ & & & $\begin{array}{l}\text { Female (age: } \\
\geq 60 \text { ) }\end{array}$ & & \\
\hline $\begin{array}{l}\mathrm{BMI}(\geq 24 \\
\mathrm{kg} / \mathrm{m} 2)\end{array}$ & $\begin{array}{l}1.083(0.813- \\
1.442)\end{array}$ & $\begin{array}{l}1.110(0.827- \\
1.490)\end{array}$ & $\begin{array}{l}\mathrm{BMI}(\geq 24 \\
\mathrm{kg} / \mathrm{m} 2)\end{array}$ & $\begin{array}{l}1.746(1.290- \\
2.172) \star \star\end{array}$ & $\begin{array}{l}1.596(1.200- \\
2.052) \star \star\end{array}$ \\
\hline $\begin{array}{l}\operatorname{BAl}(\geq \\
25.5)\end{array}$ & $\begin{array}{l}1.103(0.830- \\
1.467)\end{array}$ & $\begin{array}{l}1.070(0.796- \\
1.438)\end{array}$ & $\mathrm{BAI}(\geq 30.5)$ & $\begin{array}{l}1.395(1.177- \\
1.806) *\end{array}$ & $\begin{array}{l}1.278(1.078- \\
1.671) \star\end{array}$ \\
\hline $\begin{array}{l}W C(\geq 90 \\
\mathrm{cm})\end{array}$ & $\begin{array}{l}1.478(1.104- \\
1.980) *\end{array}$ & $\begin{array}{l}1.452(1.070- \\
1.970) *\end{array}$ & $\begin{array}{l}\text { WC }(\geq 80 \\
\mathrm{cm})\end{array}$ & $\begin{array}{l}1.703(1.226- \\
2.366) \star \star\end{array}$ & $\begin{array}{l}1.575(1.124- \\
2.208) \star \star\end{array}$ \\
\hline $\begin{array}{l}\text { WHR (> } \\
0.9)\end{array}$ & $\begin{array}{l}1.147(0.859- \\
1.530)\end{array}$ & $\begin{array}{l}1.104(0.822- \\
1.484)\end{array}$ & WHR $(>0.8)$ & $\begin{array}{l}1.637(0.855- \\
3.134)\end{array}$ & $\begin{array}{l}1.592(0.819- \\
3.093)\end{array}$ \\
\hline $\begin{array}{l}\text { WHtR }(\geq \\
0.5)\end{array}$ & $\begin{array}{l}1.225(0.916- \\
1.637)\end{array}$ & $\begin{array}{l}1.172(0.865- \\
1.588)\end{array}$ & WHtR $(\geq 0.5)$ & $\begin{array}{l}1.323(1.025- \\
1.709) \star \star\end{array}$ & $\begin{array}{l}1.203(1.023- \\
1.568) \text { * }\end{array}$ \\
\hline \multicolumn{6}{|c|}{$\begin{array}{l}\text { BMI, body mass index; BAl, body adiposity index; WC, waist circumference; WHR, waist-to-hip ratio; } \\
\text { WHtR, waist-to-height ratio. }\end{array}$} \\
\hline \multicolumn{6}{|c|}{ Model 1: unadjusted. } \\
\hline \multicolumn{6}{|c|}{$\begin{array}{l}\text { Model 2: adjusted for education, lifestyle (physical activity, smoking, alcohol drinking, sweet tooth), } \\
\text { medical history characteristics (family history or hypertension), and time of diagnosis of type } 2 \\
\text { diabetes mellitus. }\end{array}$} \\
\hline
\end{tabular}


Furthermore, we compared the area under the ROC curve (AUC) of each obesity index for the prediction of T2DM under the influence of risk factors including education, smoking, alcohol consumption, exercise, sweet tooth, hypertension and family disease history. Meanwhile, considering that after the diagnosis of T2DM, the patient may control their weight, we also considered the "time of diagnosis of T2DM" as an adjustment variable. As Table 4 shows, similar to the front results, the AUC of BAI and WC were the largest ( 0.783 and 0.614$)$ among the five anthropometric indices in two male groups. In addition, WHtR (AUC $=0.771)$ and BMI (AUC $=0.647)$ tended to be the best predictors for T2DM among females in the $18-59$ and $\geq 60$ years age groups, respectively. 
Table 4

AUCs, areas under the ROC curve for Anthropometric indices in Relation to type 2 diabetes mellitus

\section{Variable}

Variable

Male (age:18-44)

\begin{tabular}{|c|c|c|c|c|}
\hline $\mathrm{BMI}\left(\mathrm{kg} / \mathrm{m}^{2}\right)+$ other factors${ }^{*}$ & $0.748(0.677-0.819)$ & 0.683 & 0.690 & 0.373 \\
\hline BAl + other factors & $0.750(0.678-0.822)$ & 0.854 & 0.545 & 0.399 \\
\hline$W C(\mathrm{~cm})+$ other factors & $0.783(0.717-0.849)$ & 0.829 & 0.627 & 0.456 \\
\hline WHR + other factors & $0.730(0.657-0.803)$ & 0.732 & 0.635 & 0.367 \\
\hline WHtR + other factors & $0.768(0.701-0.836)$ & 0.707 & 0.667 & 0.374 \\
\hline \multicolumn{5}{|l|}{ Male (age: $\geq 60$ ) } \\
\hline $\mathrm{BMI}\left(\mathrm{kg} / \mathrm{m}^{2}\right)+$ other factors & $0.586(0.543-0.628)$ & 0.472 & 0.677 & 0.149 \\
\hline BAI + other factors & $0.585(0.542-0.629)$ & 0.439 & 0.719 & 0.158 \\
\hline$W C(\mathrm{~cm})+$ other factors & $0.614(0.572-0.657)$ & 0.593 & 0.611 & 0.204 \\
\hline WHR + other factors & $0.585(0.542-0.628)$ & 0.488 & 0.664 & 0.152 \\
\hline WHtR + other factors & $0.593(0.550-0.636)$ & 0.455 & 0.726 & 0.181 \\
\hline
\end{tabular}

Female (age:18-44)

BMI $\left(\mathrm{kg} / \mathrm{m}^{2}\right)+$ other factors

$0.769(0.709-0.829) \quad 0.936$

0.473

0.409

BAl + other factors

0.744(0.674-0.813)

0.489

0.842

0.331

WC $(\mathrm{cm})+$ other factors

$0.759(0.696-0.821)$

0.872

0.517

0.389

WHR + other factors

0.739(0.667-0.811)

0.468

0.872

0.340

WHtR + other factors

$0.771(0.712-0.831)$

0.660

0.753

0.413

Female (age: $\geq 60$ )

BMI $\left(\mathrm{kg} / \mathrm{m}^{2}\right)+$ other factors

0.647(0.612-0.682)

0.532

0.685

0.217

$\mathrm{BAl}+$ other factors

$0.624(0.587-0.660) \quad 0.451$

0.730

0.181

WC $(\mathrm{cm})+$ other factors

0.634(0.599-0.669)

0.623

0.560

0.183

WHR + other factors

0.619(0.583-0.656) $\quad 0.687$

0.482

0.169

AUC, area under curve; BMI, body mass index; BAl, body adiposity index; WC, waist circumference; WHR, waist-to-hip ratio; WHtR, waist-to-height ratio.

*Other factors: education, lifestyle (physical activity, smoking, alcohol drinking, sweet tooth), medical history characteristics (family history or hypertension), and time of diagnosis of type 2 diabetes mellitus. 


\begin{tabular}{|lcccc|}
\hline Variable & AUC $(95 \% \mathrm{Cl})$ & Sensitivity & Specificity & Youden index \\
\hline WHtR + other factors & $0.629(0.592-0.665)$ & 0.515 & 0.682 & 0.197 \\
\hline $\begin{array}{l}\text { AUC, area under curve; BMI, body mass index; BAl, body adiposity index; WC, waist circumference; } \\
\text { WHR, waist-to-hip ratio; WHtR, waist-to-height ratio. }\end{array}$ & & & \\
\hline $\begin{array}{l}* \text { Other factors: education, lifestyle (physical activity, smoking, alcohol drinking, sweet tooth), medical } \\
\text { history characteristics (family history or hypertension), and time of diagnosis of type 2 diabetes } \\
\text { mellitus. }\end{array}$ & & & \\
\hline
\end{tabular}

\section{Discussion}

In this study, we compared the associations of five anthropometric indices for T2DM among Chinese adults divided into four groups according to sex and age. Using survey data from a cross-sectional study of chronic diseases in Nantong city, east of China, we found strong evidence that there is a positive correlation between obesity-related measures and T2DM; a finding which was consistent with those of previous studies in China [15-16]. Previous studies have found that leptin level in obese people is higher than that in normal people, while adiponectin level is opposite. Leptin can activate renal sympathetic nerve and increase arterial pressure. Adiponectin can effectively protect cardiovascular system, which is negatively correlated with insulin resistance [24-25]. Obesity can lead to islet dysfunction and decrease of insulin secretion, meanwhile, adipocytes are insensitive to insulin, which leads to an increase of blood sugar [26].

The results of this study also show that, compared with other obesity indicators, WHtR had a greater impact on the risk of T2DM in the general population, which was consistent with previous research results. Previous studies have shown that height has an important influence on diabetes, while WC and WHR have not considered the influence of height [27-28]. In order to overcome this defect, most researchers used WHtR to study the relationship between overweight, obesity and chronic diseases [2930]. WHtR is easy to calculate, and has a significant relationship with waist circumference without gender difference. Taking $\geq 0.5$ as the cut-in point can well predict the risk of diabetes [31]. A study in China showed that waist-height ratio has no effect on impaired fasting blood glucose, but it is related to diabetes, which can be used as an important screening index for the elderly [32]. In addition, central and systemic obesity indices showed different correlations in different sex and age groups.

For males, WC had the highest OR and the largest AUC before after adjusting for confounding variables in both 18-59 and $\geq 60$ age groups. The results of a meta-analysis of 16 cohort studies involving different ethnic groups in Asia showed that for every additional SD in BMI and WC, the risk of diabetes was $52 \%$ and $54 \%$, respectively. WC was more closely related to diabetes [33]. A survey in China found that for every standard deviation increase in BMI and WC, the risk of diabetes increased by $53 \%$ and $64 \%$, respectively. WC had a greater impact on the risk of diabetes [34]. The conclusions of these studies were the same as those in the present study, which might be related to the fact that insulin resistance is an important pathogenic factor for diabetes [35]. Studies have confirmed that insulin antagonism is more 
obvious in central obesity, and WC is recognized as an important indicator for measuring central obesity, which can better reflect insulin resistance [36]. Therefore, it was recommended that the waist circumference can be reduced through appropriate physical exercise and other means on the basis of weight control, thereby reducing the risk of type 2 diabetes.

However, among the females, WHtR and BMI tended to be the best predictors for T2DM in the 18-59 and $\geq 60$ age groups, respectively. The reason for the sex difference in the relationship between obesity indicators and T2DM may be related to the sex difference in body fat distribution. Fat was mainly in the viscera in men and subcutaneous in women, which can provide evidence for gender differences. In thin Asian men with less subcutaneous fat, WC may be a better indicator of visceral obesity than BMI. Among thin Asian women, the impact of subcutaneous fat was greater than that of WC, and BMI may be more suitable for the indicator of overall fat and abdominal fat accumulation than WC [37-39]. On the other hand, compared with women, men were more stressed at work and slept less. Working pressure can increase the epinephrine secretion level of the body, and then lead to more fat into the abdomen [40]; Sleep deprivation was closely related to cardiovascular risk factors such as inflammatory markers in the blood circulation, and the resulting inflammatory response may be one of the mechanisms leading to metabolic diseases such as abdominal obesity and T2DM [41]. However, in recent years, Chinese women's rich diet nutrition, reduced exercise and increasing pressure at work have led to the increase of central obesity in young and middle-aged women [42]. This may explain why obesity indicators most closely associated with T2DM are inconsistent across age groups.

BAl was also included in this study as a new obesity index proposed in 2011, which was believed to be able to assess percent body fat more objectively based on hip circumference and height [43]. We found that BAI was positively associated with T2DM except in male $\geq 60$ age group. The positive association of T2DM with BAI was not stronger than that observed in other generally used obesity indices. Consistently, the capacity of BAI in predicting T2DM was also found lower than BMI, WC and WHtR, which recognized as the most frequently used obesity indices. This was inconsistent with some American research results that BAI was superior to BMI [44-45], possibly because BAI was proposed by the research on hip circumference and height of African Americans and Mexican Americans [43], while the characteristics of height and hip circumference of Chinese Han people are quite different from them.

We also noticed that there was no positive association between WHR and T2DM in all sex and age groups. Therefore, using WHR as a weight predictor for T2DM was not recommended. This finding was similar to that of a study in Changchun. In that study, the cut-off value for WHR was not suitable for predicting T2DM compared with those of BMI and WHtR [19].

One of strengths in the present study was that we randomly selected a community-based population with a broad age range; this increases the generalizability of our results to local populations. Further, we compared the predictive values of five anthropometric indices (BMI, BAI, WC, WHR, and WHtR) to achieve accurate evaluation of T2DM prediction by obesity indicators for different age and sex groups. We 
believe that the findings of the present study may provide a reference for the selection of the appropriate anthropometric obesity indices for estimating the risk of T2DM in people of different sexes and ages.

Several limitations of the present study should be considered. Firstly, as a cross-sectional study, the causality between obesity indices and T2DM cannot be properly proven. Longitudinal follow-up studies need be conducted to further verify the results of the present study. Secondly, participants who reported T2DM by themselves may alter their lifestyle habits such as diet and exercise to skew their anthropometric indices. Although this was considered and adjusted for in the model and ROC curve analyses, information migration was unavoidable. Finally, only residents from a single area were recruited for this study. Therefore, the conclusions of this study may have some limitations in extrapolation due to the diet and lifestyle differences of different regions.

\section{Conclusion}

This study suggests that there is a positive association of obesity-related anthropometric indices with T2DM in different sex and age groups. For males, WC appears to be the best anthropometric index for predicting T2DM. For 18-59 and $\geq 60$ age women, the best obesity indices related with T2DM are WHtR and $\mathrm{BMI}$, respectively.

\section{Abbreviations}

T2DM: type 2 diabetes mellitus; BMI: body mass index; BAl: body adiposity index; WC: waist circumference; WHR: waist-to-hip ratio; WHtR: waist-to-height ratio; AUC: area under the ROC curve.

\section{Declarations}

\section{Ethics approval and consent to participate}

The study scheme was approved by the Ethics Committee of the Third People's Hospital Affiliated to the School of Medicine of Nantong University. All participants obtained written informed consent prior to data collection.

\section{Consent for publication}

Not applicable.

\section{Availability of data and materials}

The data, analytic methods, and study materials will not be made available to other researchers for purposes of reproducing the results or replicating the procedure. 


\section{Competing interests}

No potential conflicts of interest relevant to this article were reported.

\section{Funding}

This work was supported by: National Chronic Disease Comprehensive Prevention and Control Demonstration Zone Construction Project and Nantong Municipal Health Commission health Promotion Project (2020JCC025).

\section{Authors' contributions}

Qiwei Ge and Min Li drafted the manuscript and analyzed the data. Zhigang Qi and Huiyan Zheng conceived of and designed the study. Zhengcheng Xu, Yuxin Cao, Hao Huang and Xiaoyang Duan contributed to the acquisition of the data. Xun Zhuang revised the manuscript. All authors read and approved the final manuscript.

\section{Acknowledgments}

The authors gratefully acknowledge the support of the study participants, study staff, and partner organizations participating in the baseline survey and follow-up investigation.

\section{References}

1. Saeedi P, Petersohn I, Salpea P, Malanda B, Karuranga S, Unwin N, et al. Global and regional diabetes prevalence estimates for 2019 and projections for 2030 and 2045: Results from the International Diabetes Federation Diabetes Atlas, 9th edition. Diabetes Res Clin Pract. 2019 Nov;157:107843.

2. Wang C, Li J, Xue H, Li Y, Huang J, Mai J, et al. Type 2 diabetes mellitus incidence in Chinese: contributions of overweight and obesity. Diabetes Res Clin Pract. 2015 Mar;107(3):424-32

3. Yang W, Lu J, Weng J, Jia W, Ji L, Xiao J, et al. Prevalence of diabetes among men and women in China. N Engl J Med. 2010 Mar 25;362(12):1090-101.

4. Lu B, Yang Y, Yang Z, Feng X, Wang X, Zhang Z, Hu R. Insulin resistance in Chinese patients with type 2 diabetes is associated with $\mathrm{C}$-reactive protein independent of abdominal obesity. Cardiovasc Diabetol. 2010 Dec 19;9:92.

5. Lean MEJ, Leslie WS, Barnes AC, Brosnahan N, Thom G, McCombie L, et al. Durability of a primary care-led weight-management intervention for remission of type 2 diabetes: 2-year results of the DiRECT open-label, cluster-randomised trial. Lancet Diabetes Endocrinol. 2019 May;7(5):344-355. 
6. Bennett DA, Du H, Bragg F, Guo Y, Wright N, Yang L, et al. Physical activity, sedentary leisure-time and risk of incident type 2 diabetes: a prospective study of 512000 Chinese adults. BMJ Open Diabetes Res Care. 2019 Dec 18;7(1):e000835.

7. Mohanty SA, Woolhandler S, Himmelstein DU, Bor DH. Diabetes and cardiovascular disease among Asian Indians in the United States. J Gen Intern Med. 2005;20(5):474-478.

8. Yang J, Wang F, Wang J, Han X, Hu H, Yu C, et al. Using different anthropometric indices to assess prediction ability of type 2 diabetes in elderly population: a 5 year prospective study. BMC Geriatr. 2018 Sep 17;18(1):218.

9. Lim RBT, Chen C, Naidoo N, Gay G, Tang WE, Seah D, et al. Anthropometrics indices of obesity, and all-cause and cardiovascular disease-related mortality, in an Asian cohort with type 2 diabetes mellitus. Diabetes Metab. 2015 Sep;41(4):291-300.

10. Hlatky MA, Chung SC, Escobedo J, Hillegass WB, Melsop K, Rogers W, et al. The effect of obesity on quality of life in patients with diabetes and coronary artery disease. Am Heart J. 2010 Feb;159(2):292-300.

11. Motamed N, Rabiee B, Keyvani H, Hemasi GR, Khonsari M, Saeedian FS, et al. The Best Obesity Indices to Discriminate Type 2 Diabetes Mellitus. Metab Syndr Relat Disord. 2016 Jun;14(5):249-53.

12. Zerga AA, Bezabih AM, Adhanu AK, Tadesse SE. Obesity Indices for Identifying Metabolic Syndrome Among Type Two Diabetes Patients Attending Their Follow-Up in Dessie Referral Hospital, North east Ethiopia. Diabetes Metab Syndr Obes. 2020 Apr 23;13:1297-1304.

13. Wang S, Ma W, Yuan Z, Wang SM, Yi X, Jia H, Xue F. Association between obesity indices and type 2 diabetes mellitus among middle-aged and elderly people in Jinan, China: a cross-sectional study. BMJ Open. 2016 Nov 3;6(11):e012742. doi: 10.1136/bmjopen-2016-012742. PMID: 27810975; PMCID: PMC5129047.

14. Xiao X, Liu Y, Sun C, Gang X, Cheng J, Tian S, et al. Evaluation of different obesity indices as predictors of type 2 diabetes mellitus in a Chinese population. J Diabetes. 2015 May;7(3):386-92.

15. Rezende FA, Ribeiro AQ, Mingoti SA, Pereira PF, Marins JC, Priore SE, Franceschini SC. Anthropometric patterns of adiposity, hypertension and diabetes mellitus in older adults of Viçosa, Brazil: A population-based study. Geriatr Gerontol Int. 2018 Apr;18(4):584-591. doi: 10.1111/ggi.13219. Epub 2018 Jan 2. PMID: 29292569.

16. de Oliveira CM, Pavani J, Krieger JE, de Oliveira Alvim R, Mourão-Junior CA, da Costa Pereira A. Body adiposity index in assessing the risk of type 2 diabetes mellitus development: the Baependi Heart Study. Diabetol Metab Syndr. 2019 Aug 29;11:76.

17. Wei J, Liu X, Xue H, Wang Y, Shi Z. Comparisons of Visceral Adiposity Index, Body Shape Index, Body Mass Index and Waist Circumference and Their Associations with Diabetes Mellitus in Adults. Nutrients. 2019 Jul 12;11(7):1580.

18. Bragg F, Tang K, Guo Y, lona A, Du H, Holmes MV, et al. Associations of General and Central Adiposity With Incident Diabetes in Chinese Men and Women. Diabetes Care. 2018 Mar;41(3):494-502. doi: 10.2337/dc17-1852. Epub 2018 Jan 3. PMID: 29298802; PMCID: PMC6548563. 
19. Wang Xiaofeng, Ji Xinge. Sample Size Estimation in Clinical Research: From Randomized Controlled Trials to Observational Studies. Chest. 2020;158:S12-S20.

20. Ma RCW. Epidemiology of diabetes and diabetic complications in China. Diabetologia. 2018;61(6):1249-1260.

21. Chinese Guidelines for the Prevention and treatment of Type 2 diabetes (2017 edition). Chinese Journal of Diabetes. 2015;10(01):4-67.

22. Chen $\mathrm{CM}$, Kong LZ. Chinese guidelines for the prevention and control of overweight and obesity in adults. Beijing: People's medical publishing house, 2006:3.

23. Keli Yu, Shan Li, LianBing Zheng, Yonglan Li, Jinping Bao, Xinghua Zhang. Research on the applicability of BAI to obesity evaluation of Chinese Rural Han Adults. Journal of Tianjin Normal University (natural science edition). 2019;33 (05):70-75.

24. Kita S, Maeda N, Shimomura I. Interorgan communication by exosomes, adipose tissue, and adiponectin in metabolic syndrome. J Clin Invest. 2019 Oct 1;129(10):4041-4049.

25. Henneman P, Janssens AC, Zillikens MC, Frolich M, Frants RR, Oostra BA, et al. Menopause impacts the relation of plasma adiponectin levels with the metabolic syndrome. J Intern Med. 2010 Apr;267(4):402-9.

26. Kahn Steven E, Hull Rebecca L, Utzschneider Kristina M. Mechanisms linking obesity to insulin resistance and type 2 diabetes. Nature. 2006;444:840-846.

27. Wickramasinghe VP, Arambepola C, Bandara P, et al. Insulin resistance in a cohort of 5-15 year old children in urban Sri Lanka. BMC Res Notes. 2017;10(1):347.

28. Wickramasinghe VP, Arambepola C, Bandara P, Abeysekera M, Kuruppu S, Dilshan P, Dissanayake BS. Insulin resistance in a cohort of 5-15 year old children in urban Sri Lanka. BMC Res Notes. $2017 \mathrm{Jul}$ 28;10(1):347.

29. Dong B, Wang Z, Arnold LW, Song Y, Wang HJ, Ma J. Simplifying the screening of abdominal adiposity in Chinese children with waist-to-height ratio. Am J Hum Biol. 2016 Nov;28(6):945-949.

30. Tarleton HP, Smith LV, Zhang ZF, Kuo T. Utility of anthropometric measures in a multiethnic population: their association with prevalent diabetes, hypertension and other chronic disease comorbidities. J Community Health. 2014 Jun;39(3):471-9.

31. He Y, Zhai F, Ma G, Feskens EJ, Zhang J, Fu P, Van't Veer P, Yang X. Abdominal obesity and the prevalence of diabetes and intermediate hyperglycaemia in Chinese adults. Public Health Nutr. 2009 Aug;12(8):1078-84.

32. Yuan Y, Xie H, Sun L, Wang B, Zhang L, Han H, Yao R, Sun Y, Fu L. A Novel Indicator of Children's Lipid Accumulation Product Associated with Impaired Fasting Glucose in Chinese Children and Adolescents. Diabetes Metab Syndr Obes. 2020 May 14;13:1653-1660.

33. Nyamdorj R, Qiao Q, Söderberg S, Pitkäniemi JM, Zimmet PZ, Shaw JE, et al. BMI compared with central obesity indicators as a predictor of diabetes incidence in Mauritius. Obesity (Silver Spring). 2009 Feb;17(2):342-8. 
34. Karter AJ, D'Agostino RB Jr, Mayer-Davis EJ, Wagenknecht LE, Hanley AJ, Hamman RF, et al. Abdominal obesity predicts declining insulin sensitivity in non-obese normoglycaemics: the Insulin Resistance Atherosclerosis Study (IRAS). Diabetes Obes Metab. 2005 May;7(3):230-8.

35. Gray RS, Fabsitz RR, Cowan LD, Lee ET, Howard BV, Savage PJ. Risk factor clustering in the insulin resistance syndrome. The Strong Heart Study. Am J Epidemiol. 1998;148(9):869-878.

36. Caspard H, Jabbour S, Hammar N, Fenici P, Sheehan JJ, Kosiborod M. Recent trends in the prevalence of type 2 diabetes and the association with abdominal obesity lead to growing health disparities in the USA: An analysis of the NHANES surveys from 1999 to 2014. Diabetes Obes Metab. 2018;20(3):667-671.

37. Alokail MS, Al-Daghri NM, Al-Attas OS, Hussain T. Combined effects of obesity and type 2 diabetes contribute to increased breast cancer risk in premenopausal women. Cardiovasc Diabetol. 2009 Jun 23;8:33.

38. Won KB, Hur SH, Cho YK, Yoon HJ, Nam CW, Kim KB, et al. Comparison of 2-year mortality according to obesity in stabilized patients with type 2 diabetes mellitus after acute myocardial infarction: results from the DIAMOND prospective cohort registry. Cardiovasc Diabetol. 2015 Oct 15;14:141.

39. Tchernof A, Després JP. Pathophysiology of human visceral obesity: an update. Physiol Rev. 2013;93(1):359-404.

40. Wan H, Wang Y, Xiang Q, Fang S, Chen Y, Chen C, et al. Associations between abdominal obesity indices and diabetic complications: Chinese visceral adiposity index and neck circumference. Cardiovasc Diabetol. 2020 Jul 31;19(1):118.

41. Suarez EC. Self-reported symptoms of sleep disturbance and inflammation, coagulation, insulin resistance and psychosocial distress: evidence for gender disparity. Brain Behav Immun. 2008;22(6):960-968.

42. Zh Zhang JG, Wang ZH, Wang HJ, Du WW, Su C, Zhang J, et al. Dietary patterns and their associations with general obesity and abdominal obesity among young Chinese women. Eur $\mathrm{J}$ Clin Nutr. 2015 Sep;69(9):1009-14.

43. Bergman RN. A better index of body adiposity. Obesity (Silver Spring). 2012 Jun;20(6):1135.

44. Schulze MB, Thorand B, Fritsche A, Häring HU, Schick F, Zierer A, et al. Body adiposity index, body fat content and incidence of type 2 diabetes. Diabetologia. 2012 Jun;55(6):1660-7.

45. Freedman DS, Thornton JC, Pi-Sunyer FX, Heymsfield SB, Wang J, Pierson RN Jr, Blanck HM, Gallagher D. The body adiposity index (hip circumference $\div$ height(1.5)) is not a more accurate measure of adiposity than is BMl, waist circumference, or hip circumference. Obesity (Silver Spring). 2012 Dec;20(12):2438-44.

\section{Figures}




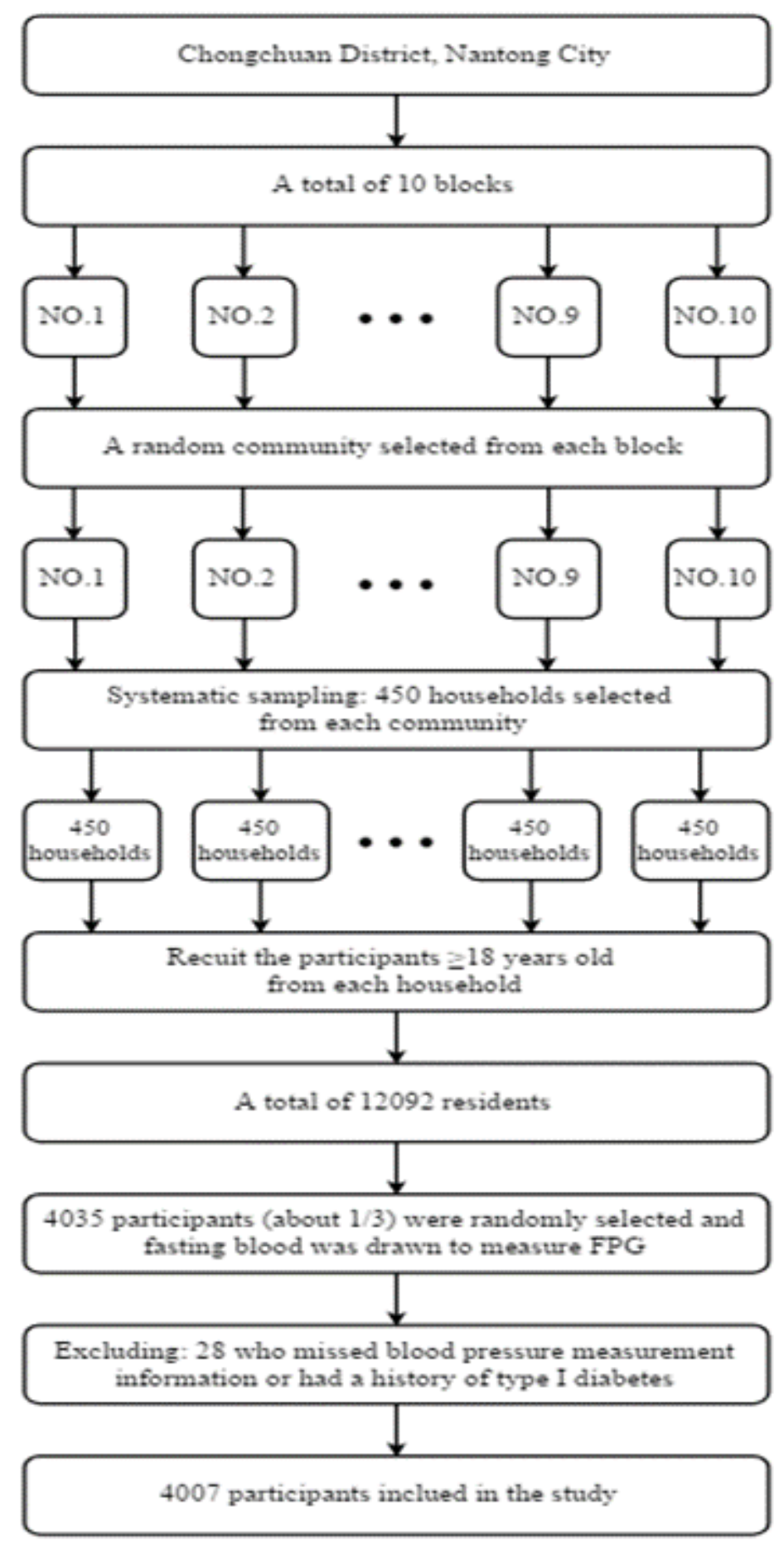

\section{Figure 1}

The sample diagram of the study object 